\title{
Multi-Layer Onlay Graft Using Hydroxyapatite Cement Placement without Cerebrospinal Fluid Diversion for Endoscopic Skull Base Reconstruction
}

\author{
Young-Hoon Kim, ${ }^{1}$ Ho Kang, ${ }^{2}$ Yun-Sik Dho, ${ }^{3}$ Kihwan Hwang, ${ }^{4}$ Jin-Deok Joo, ${ }^{5}$ Yong Hwy Kim ${ }^{2}$ \\ Department of Neurological Surgery, Asan Medical Center, University of Ulsan College of Medicine, Seoul, Korea \\ Department of Neurosurgery, ${ }^{2}$ Seoul National University Hospital, Seoul, Korea \\ Department of Neurosurgery, ${ }^{3}$ Chungbuk National University Hospital, Cheongju, Korea \\ Department of Neurosurgery, ${ }^{4}$ Seoul National University Bundang Hospital, Seongnam, Korea \\ Department of Neurosurgery, Jeju National University Hospital, Jeju, Korea
}

Objective : The skull base reconstruction step, which prevents cerebrospinal fluid (CSF) leakage, is one of the most challenging steps in endoscopic skull base surgery (ESS). The purpose of this study was to assess the outcomes and complications of a reconstruction technique for immediate CSF leakage repair using multiple onlay grafts following ESS.

Methods : A total of 230 consecutive patients who underwent skull base reconstruction using multiple onlay grafts with fibrin sealant patch (FSP), hydroxyapatite cement (HAC), and pedicled nasoseptal flap (PNF) for high-flow CSF leakage following ESS at three institutions were enrolled. We retrospectively reviewed the medical and radiological records to analyze the preoperative features and postoperative results.

Results : The diagnoses included craniopharyngioma (46.8\%), meningioma (34.0\%), pituitary adenoma (5.3\%), chordoma (1.6\%), Rathke's cleft cyst (1.1\%) and others ( $n=21,11.2 \%)$. The trans-planum/tuberculum approach (94.3\%) was the most commonly adapted surgical method, followed by the trans-sellar and transclival approaches. The third ventricle was opened in 78 patients (41.5\%). Lumbar CSF drainage was not performed postoperatively in any of the patients. Postoperative CSF leakage occurred in four patients $(1.7 \%)$ due to technical mistakes and were repaired with the same technique. However, postoperative meningitis occurred in $13.5 \%(n=31)$ of the patients, but no microorganisms were identified. The median latency to the diagnosis of meningitis was 8 days (range, 2-38). CSF leakage was the unique risk factor for postoperative meningitis $(p<0.001)$.

Conclusion : The use of multiple onlay grafts with FSP, HAC, and PNF is a reliable reconstruction technique that provides immediate and complete CSF leakage repair and mucosal grafting on the skull base without the need to harvest autologous tissue or perform postoperative CSF diversion. However, postoperative meningitis should be monitored carefully.

Key Words : Skull base neoplasms · Cerebrospinal fluid leak · Hydroxyapatite cement · Endoscopy.

- Received : August 11, 2020 •Revised : September 29, 2020 •Accepted : October 7, 2020

- Address for reprints : Yong Hwy Kim

Department of Neurosurgery, Seoul National University Hospital, 101 Daehak-ro, Jongno-gu, Seoul 03080, Korea

Tel : +82-2-2072-4062, Fax : +82-2-744-8459, E-mail : kimyh96@snu.ac.kr, ORCID : ORCID : https://orcid.org/0000-0001-9009-4191

This is an Open Access article distributed under the terms of the Creative Commons Attribution Non-Commercial License (http://creativecommons.org/licenses/by-nc/4.0) which permits unrestricted non-commercial use, distribution, and reproduction in any medium, provided the original work is properly cited. 


\section{INTRODUCTION}

Advances in anatomical knowledge and surgical techniques have expanded the indications of endoscopic skull base surgery (ESS) in recent decades. The favorable results that have been documented regarding tumor control and neurologic outcomes have contributed to the increased interest in ESS, and ESS is recognized not as an alternative surgical technique but instead as one of surgical approaches that should be considered $^{36,38,51,55)}$.

The repair of cerebrospinal fluid (CSF) leakage is one of the most pressing concerns, even with the microscopic transsphenoidal approach (TSA). With the significant expansion of the surgical windows with the introduction of ESS, CSF leakage is caused by not only a tear in the diaphragma sellae but also by the wide opening of the basal cisterns or the third ventricle. Therefore, different reconstruction concepts and techniques for high-flow CSF leakage through large skull base defects are required. A grading system was proposed and updated to identify an optimally designed reconstruction strategy, and various reconstruction techniques have been introduced $^{13,18,32,42,63)}$. A review on endoscopic skull base reconstruction for large dural defects showed a CSF leakage rate as high as $11.5 \%{ }^{33)}$. It is evident that the CSF leakage rates dramatically decreased after the introduction of the vascularized flap. Currently, various combinations of reconstruction techniques with a multilayer method have been adopted on an individual basis, and the vascularized mucosal flap continues to be beneficial. However, the vascularized mucosal flap does not provide immediate watertight leakage repair and requires postoperative CSF diversion, such as lumbar drainage, for a better success rate ${ }^{64)}$. The more serious concern is that skull base reconstruction can fail even with multilayer reconstruction, including the use of the pedicled flap following CSF diversion, after several days. Lumbar drainage, which is mostly used with CSF diversion after ESS, usually requires the restriction of postoperative ambulation and sometimes causes longer hospital stays and complications, such as deep vein thrombosis (DVT), pulmonary complications, and intracranial events related to intracranial hypotension ${ }^{8,64)}$.

To our knowledge, there is no surgical technique that achieves an immediate complete repair of high-flow CSF leakage without CSF diversion, except for direct sutures. We introduce a surgical reconstruction technique with artificial materials and a pedicled mucosal flap for immediate watertight skull base reconstruction and retrospectively analyzed the clinical results.

\section{MATERIALS AND METHODS}

\section{Patients}

This study was approved by the Institutional Review Board of Seoul Natinoal University Hospital, Seoul Natinoal Uniersity Bundang Hospital, and Asan Medical Center (No. H-1910-061-1071) and included patients who underwent multiple onlay reconstruction with fibrin sealant patch (FSP), hydroxyapatite cement (HAC), and pedicled nasoseptal flap (PNF) for grade 3 high flow CSF leakage following ESS for intracranial tumors from October 2012 to October $2018^{13)}$. Therefore, we excluded patients who had grade 1 or 2 CSF leakage by intradural extraarachnoid tumors, such as usual pituitary adenomas. Clinical data encompassing the preoperative demographic details, pathological results, CSF leakage, and postoperative infection were retrospectively collected and interpreted based on the surgical approaches. Magnetic resonance (MR) images with gadolinium contrast and nasal cavity computed tomography (CT) images were verified in all patients before surgery to elucidate the details of the intracranial anatomy and individual variations of the nasal cavity. Brain CT scans with $1 \mathrm{~mm}$ slices were performed immediately after the surgery to identify the state of rigid reconstruction and intracranial hemorrhage, and postoperative MR images were acquired within 48 hours after surgery.

Preoperative neuroendocrinological evaluations were dependent on the presumptive diagnosis by the imaging features and the tumor locations. In parasellar tumors, basal hormones, including serum thyroid-stimulating hormone, free T4, adrenocorticotropic hormone, cortisol, prolactin, growth hormone, insulin-like growth factor-1, luteinizing hormone, follicle-stimulating hormone, estradiol, progesterone, and total testosterone, were measured from the serum sampled in the early morning. A rapid adrenocorticotropic hormone stimulation test was performed in the suspected patients. The visual field defects were evaluated with Goldmann or Humphrey perimetry. The same items of the preoperative evaluation were performed again in the follow-up 3 months after surgery. 


\section{Indications}

We adapted our multiple onlay watertight reconstruction techniques to reconstruct high-flow CSF leakage (grade 3) after ESS in patients where all boundaries of the surgical window were surrounded by rigid the skull base bone with reliable hardness and a sufficient width to anchor the HCA and graft the PNF reliably ${ }^{13)}$. As seen in the lower clivus approach or trans-odontoid approaches, the surgical windows surrounded by any soft tissue, regardless of size, were contraindi- cated (Fig. 1). An eroded or thin surrounding skull base bone, which is typically observed in pituitary adenomas with sellar enlargement, was also repaired with other reconstruction techniques. We also avoided this technique in patients with excessive bloody or wet surgical environments, as these environments make it difficult to achieve a watertight seal in the attachment of the grafts and might cause a crack in the HAC during the hardening process.
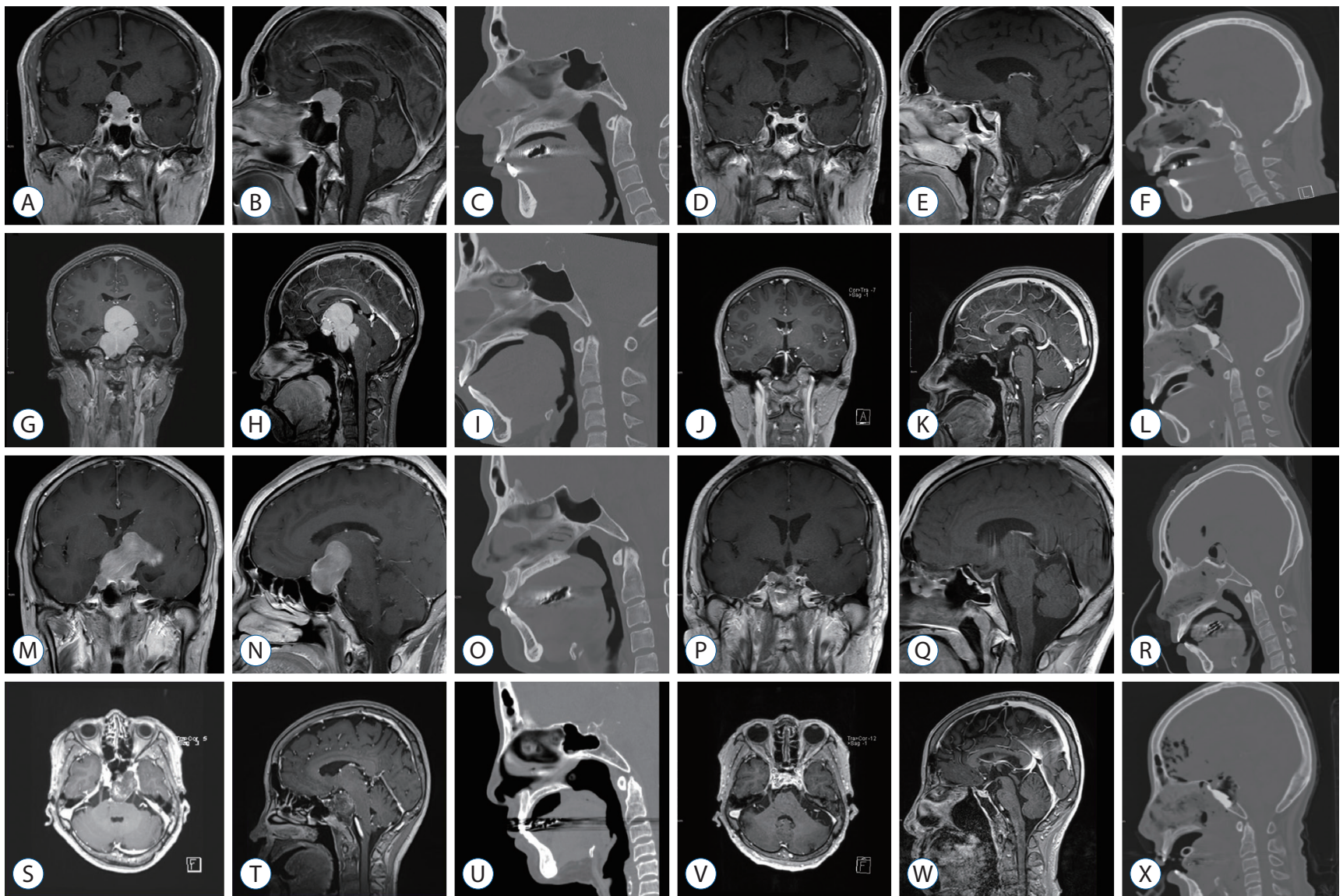

Fig. 1. Preoperative $M R$ images $(A, B, G, H, M, N, S$, and $T)$ and $C T$ scans $(C, I, O$, and $U)$. The postoperative $M R$ images $(D, E, J, K, P, Q, V$, and $W)$ showed no artifacts due to the reconstruction The immediate postoperative $C T$ scan $(F, L, R$, and $X)$. The extent of the HAC covering the skull base bone could be evaluated with the immediate postoperative $C T$ scans ( $F, L, R$, and X). A-F : Tuberculum sellae meningioma. G-L : Craniopharyngioma. M-R : Pituitary adenoma. S-X : Chordoma. Pre- and post-operative MR images and CT scans of representative diseases. The postoperative MR images showed the wellenhanced PNF and no artifact by the reconstruction materials. The postoperative CT scans is useful to evaluate the extent of the HAC covering the skull base bone. A-F : Preoperative MR images ( $A$ and $B$ ) and $C T$ scan $(C)$ of tuberculum sellae meningioma with postoperative MR images (D and E) and CT scans (F) after trans-tuberculum approach. G-L : Preoperative MR images ( $\mathrm{G}$ and H) and CT scan (I) of craniopharyngioma. The postoperative MR images ( $\mathrm{J}$ and $\mathrm{K}$ ) and $\mathrm{CT}$ scans $(\mathrm{L})$ showed the well-reconstructed skull base after trans-tuberculum and trans-clivus approach with resection of dorsum sellae. $M-R$ : Preoperative MR images ( $M$ and N) of giant pituitary adenoma with lateral extension. The preoperative $C T$ scan (O) showed the preserved tuberculum and sellar floor. Postoperative MR images ( $P$ and $Q$ ) and CT scan (R) showed the clear sphenoid sinus and surgical window of trans-sella approach. S-X : Preoperative MR images (S and T) and CT scans (U) showed the displaced pituitary gland upwardly and destructed clivus and dorsum sellae by the tumor. Postoperative MR images and CT scans (V-X) showed the normally located pituitary gland with the rigid support by the HAC. MR : magnetic resonance, $\mathrm{CT}$ : computed tomography, $\mathrm{HAC}$ : hydroxyapatite cement. 


\section{Surgical procedures}

ESS procedures were performed with an image-guided neuronavigation system, and endonasal corridors were adopted ${ }^{29)}$. Sufficient sphenoidotomy was performed following storage of the pedicled nasoseptal mucosal flap in the choana. The thin skull base mucosa was removed by approximately $1 \mathrm{~cm}$ more than the target skull base bone was, which was drilled to secure a dry grafting site for the multiple onlay grafts achieved by the spontaneous hemostasis of denuded bone during subsequent surgical procedures. Following the individually designed plan for drilling the skull base bone, the midline vertical dural incision was made first, and a bilateral tack-up suture for each dural margin was pulled through each nostril. Then, a horizontal dural incision was made at the end of the vertical incision line and expanded to the lateral end of the exposed dura. The pulled dural window served as a protective barrier for the surgical window from the blood coming from the sphenoid or from between the dural and skull base bone during the operation. The tumor was always dissected and removed with microsurgical techniques, and the details were not different from those in previous reports ${ }^{14,36,38,54)}$. The loose approximation of bilateral dura after the removal of the tackup suture was the first step of the reconstruction process. Then, a rectangular shaped FSP (TachoSil ${ }^{\circledR}$; Takeda Pharma, Linz, Austria) measuring $2.5 \times 1.5 \mathrm{~cm}$ was attached on the denuded skull base around the bony defect with gentle compression and a cottonoid patty. The additional FSPs slightly overlapped with the previous FSP and were attached to the bony window margin. The sphenoid sinus and nasal corridor were packed with cottonoid patties soaked in $1: 10000$ epinephrine after the complete sealing of the bony defect with FSPs to secure the dry state of the reconstruction site field from the blood coming from the mucosa. After removal of packed cottonoid patties, the FSPs and the exposed skull base bone around the FSPs were completely covered by the injection of HAC paste (HydroSet ${ }^{\circledR}$; Stryker, Kalamazoo, MI, USA) in the dry state. To avoid a defect or dehiscence of the injected HAC from the skull base, we started to inject the HAC from the border of the attached FSPs and then injected it on the FSPs. The packing of diluted epinephrine soaked pledgets with gentle suction over the HAC was helpful in preventing a crack in the HAC during the hardening process by the blood coming from the nasal cavity. The solidification of the HAC paste with sufficient hardness was confirmed by a gentle touch ap- proximately 3 to 5 minutes later. We covered the HAC with PNF and verified that the margin of the PNF was completely
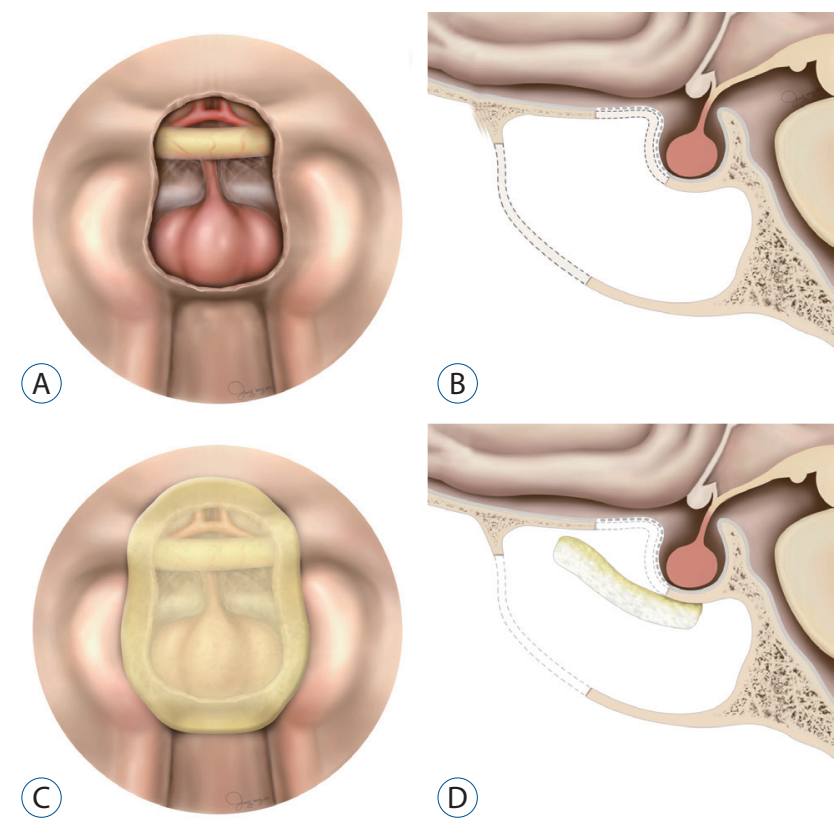

(D)
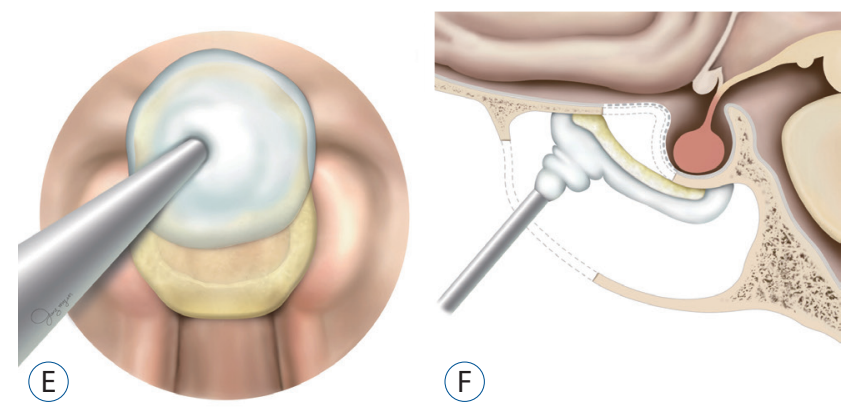

(F)
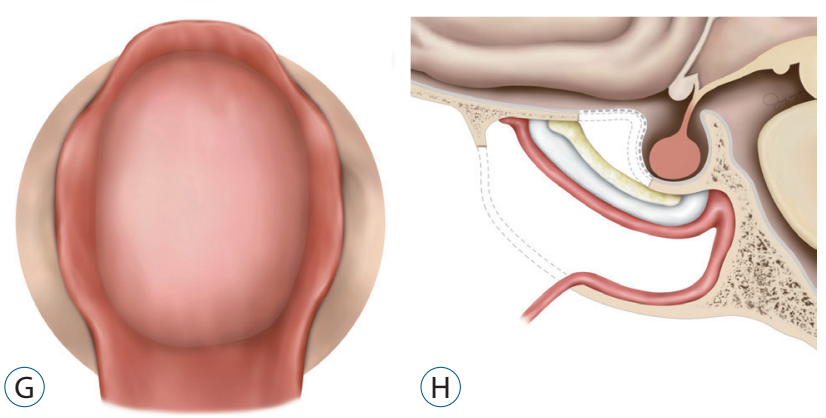

(H)

Fig. 2. Illustrations for steps of the multiple onlayers watertight reconstruction in trans-tuberculum approaches. A and $B$ : Intraoperative (A) and sagittal $(B)$ view of the skull base defect. $C$ and D : The complete onlayer sealing of bony defect with FSPs achieved a temporary watertight barrier. E and F : The HAC were injected and layered on the FSPs and surrounding denuded skull base bone along the margin of the FSP. G and $\mathrm{H}$ : The consolidated HAC in the dry state was covered by the PNF, and the attachment of the PNF to the denuded bone around the HAC was confirmed. FSP : fibrin sealant patch, HAC : hydroxyapatite cement, PNF : pedicled nasoseptal flap. 
grafted on the denuded skull base. Keeping the dry state of the skull base was an important aspect in achieving a completely watertight graft of the multiple onlay materials. We applied oxidized cellulose (Surgicel ${ }^{\circledR}$; Johnson and Johnson, New Brunswick, NJ, USA) and hydrogel sealant (DuraSeal ${ }^{\circledR}$; Confluent Surgical, Inc., Waltham, MA, USA) to prevent the migration of the PNF after surgery. Then, we sutured the thin silastic sheet to the nasal septum to prevent the development of a crust on the flap harvesting site and synechia between the turbinates and septum. The nasal cavity was packed with the absorbable nasal dressing material (Nasopore ${ }^{\circledR}$; Stryker) to prevent epistaxis from the oozed blood and mucosal synechia by mucosal swelling (Fig. 2 and Supplementary Video 1).

\section{Postoperative care and evaluations}

There were no limitations in ambulation or coughing after surgery, and we encouraged walking for postoperative lung care and the prevention of DVT. The first endoscopic nasal cavity evaluation was scheduled on the second postoperative day. The packed materials in the nasal cavity that prevented epistaxis from the swelled mucosa were removed, and then the reconstructed skull base was observed with an endoscope to verify the viability of PNF and CSF leakage. We recommended that normal saline mist be pumped frequently into the nasal cavity to prevent a crust from forming on the silastic sheets sutured on the bilateral septal mucosa. We monitored the general medical status, such as fever and serum electrolyte imbalance, which occurs with diabetes insipidus, for at least 5 days and then discharged the patients. Routine nasal cavity examinations were scheduled at 1,2, 4, and 12 weeks after the surgery. The silastic sheets were usually removed 1 month postoperatively after complete healing of the PNF harvest site was observed. An endoscopic nasal examination was performed promptly to identify CSF leakage in patients with a new-onset headache, febrile sensation or any minor meningeal irritation signs. Then, the lumbar puncture for the CSF examination was performed to determine whether CSF leakage occurred. Meningitis was diagnosed when the cell index was over 4 because a diagnosis in the early stage of infection can be missed due to the surgical debris. Empirical antibiotics were prescribed before the CSF culture results were obtained and then the antibiotics were modified to target the isolated microorganism.

\section{Data analysis}

The demographical features, pathological entities, surgical approaches and complications were descriptively analyzed. We attempted to identify the risk factor for postoperative infection using Fisher's exact test. We used SPSS software (version 24.0; IBM, Armonk, NY, USA) for data collection and statistical analyses.

\section{RESULTS}

\section{Patients}

This study enrolled 230 patients who underwent skull base reconstruction with multiple onlay techniques without CSF diversion after EEA from three institutions. The median age and follow-up period were 48.0 years old (range, 14-76) and 25.5 months (range, 1-73), respectively. Craniopharyngiomas were the most common pathologic entity, followed by meningiomas and pituitary adenomas. All pituitary adenomas were giant or supraglandular ${ }^{54}$. Other pathologic entities included germinomas, optic gliomas, epidermoid cysts, gangliogliomas, pilocytic astrocytomas of the hypothalamus, pituicytomas, and atypical teratoid rhabdoid tumors. The goal of surgery was a pathologic diagnosis in cases with germinomas and optic gliomas because of atypical clinical presentations or imaging findings. The demographic data and pathological entities by the surgical approaches are summarized in Table 1.

Preoperative hydrocephalus was found in 36 patients (15.7\%), and 44 patients (19.1\%) underwent ESS for recurrent tumors. Postoperative steroids were prescribed for more than 1 week in 150 patients $(65.2 \%)$.

\section{Skull base reconstruction}

None of the patients underwent CSF diversion, such as lumbar drainage, after surgery. Postoperative leakage was found in four patients $(1.7 \%)$ through a pinpoint sized gap between the HAC and skull base. This leakage was caused by an incomplete seal in the margins of the FSPs with HAC. The crack of HAC, caused by the wet state in applying procedure, was found in one case. Flap necrosis also occurred in one of the patients. All defects were removed completely, and the skull base was repaired by the same technique with reuse of the PNF in three patients. Postoperative meningitis was diagnosed in 31 patients (13.5\%), and no microorganisms were cultured. 
Table 1. Demographic and pathological details by surgical approaches

\begin{tabular}{|c|c|c|c|c|c|}
\hline & $\begin{array}{l}\text { Trans-planum/ } \\
\text { tuberculum }(n=217)\end{array}$ & $\begin{array}{l}\text { Trans-sella } \\
\quad(n=5)\end{array}$ & $\begin{array}{l}\text { Trans-clivus } \\
(n=4)\end{array}$ & $\begin{array}{l}\text { Trans-tuberculum, } \\
\text { sella and clivus } \\
\qquad(n=4)\end{array}$ & $\begin{array}{c}\text { Total } \\
(n=230)\end{array}$ \\
\hline Female & $123(56.7)$ & $2(40.0)$ & $4(100.0)$ & $2(50.0)$ & $131(57.0)$ \\
\hline Age (years) & $47.2 \pm 15.4$ & $42.0 \pm 4.4$ & $51.3 \pm 16.4$ & $29.3 \pm 18.4$ & $46.8 \pm 5.5$ \\
\hline Follow-up period (months) & $29.7 \pm 21.4$ & $15.4 \pm 12.5$ & $26.3 \pm 13.0$ & $28.8 \pm 7.1$ & $29.3 \pm 21.0$ \\
\hline \multicolumn{6}{|l|}{ Pathologies } \\
\hline Craniopharyngioma & 99 (45.6) & $1(20.0)$ & $0(0.0)$ & $3(75.0)$ & $103(44.8)$ \\
\hline Meningioma & $71(32.7)$ & $0(0.0)$ & $1(25.0)$ & $0(0.0)$ & $72(31.3)$ \\
\hline Pituitary adenoma & $19(8.8)$ & $3(60.0)$ & $0(0.0)$ & $0(0.0)$ & $22(9.6)$ \\
\hline Rathke's cleft cyst & $7(3.2)$ & $0(0.0)$ & $0(0.0)$ & $0(0.0)$ & $7(3.0)$ \\
\hline Chordoma & $0(0.0)$ & $0(0.0)$ & $3(75.0)$ & $1(25.0)$ & $4(1.7)$ \\
\hline Others & $21(9.7)$ & $1(20.0)$ & $0(0.0)$ & $0(0.0)$ & $22(9.6)$ \\
\hline
\end{tabular}

Values are presented as mean \pm standard deviation or number (\%)

Table 2. CSF leakage and postoperative infection

\begin{tabular}{|c|c|c|}
\hline & CSF leakage & Infection \\
\hline \multicolumn{3}{|l|}{ Institutions } \\
\hline A & $1 / 188(0.5)$ & 25/188 (13.3) \\
\hline B & $2 / 36(5.6)$ & 4/36 (11.1) \\
\hline C & $1 / 6(16.7)$ & $2 / 6(33.3)$ \\
\hline \multicolumn{3}{|l|}{ Pathologies } \\
\hline Craniopharyngioma & $3 / 103(2.9)$ & 19/103 (18.4) \\
\hline Meningioma & $0 / 72(0.0)$ & $9 / 72(12.5)$ \\
\hline Pituitary adenoma & $1 / 22(4.5)$ & $2 / 22(9.1)$ \\
\hline Rathke's cleft cyst & $0 / 7(0.0)$ & $0 / 7(0.0)$ \\
\hline Chordoma & $0 / 4(0.0)$ & 0/4 (0.0) \\
\hline Others & $0 / 22(0.0)$ & $1 / 22(4.5)$ \\
\hline \multicolumn{3}{|l|}{ Surgical corridors } \\
\hline Trans-planum/tuberclum & 4/217 (1.8) & $30 / 217(13.8)$ \\
\hline Trans-sella & $0 / 5(0.0)$ & $0 / 5(0.0)$ \\
\hline Trans-clivus & $0 / 4(0.0)$ & $1 / 4(25.0)$ \\
\hline Trans-tuberculum, sella and clivus & $0 / 4(0.0)$ & - \\
\hline \multicolumn{3}{|l|}{ 3rd ventricle opening } \\
\hline Not opened & $0 / 143(0.0)$ & $15 / 143(10.5)$ \\
\hline Opened & $4 / 83(4.6)$ & $16 / 87(18.4)$ \\
\hline Overall & $4 / 230(1.7)$ & $31 / 230(13.5)$ \\
\hline
\end{tabular}

Values are presented as n/N (\%). CSF : cerebrospinal fluid

The median and mean time to diagnosis of meningitis from surgery was 7.5 and 11.0 days (range, 2-38), respectively. No sequela remained after the management of meningitis. The only risk factor for meningitis was CSF leakage $(p<0.001)$. CSF leakage and postoperative infection are grouped with the clinical factors in Table 2. There were no complications, such as 
atelectasis or DVT, related to immobilization. All PNFs, except one with flap necrosis, were well engrafted on the onlay HAC and denuded skull base bone, showing complete healing of skull base mucosa.

\section{DISCUSSION}

In past decades, ESS has been applied in more in-depth anatomical studies and has involved more advancements in optical and instrumental technologies. It cannot be denied that the introduction of the PNF was one of the most important steps for the popularization of ESS as an innovative solution for skull base reconstruction ${ }^{24,35)}$. Currently, multi-layer reconstruction using various materials and techniques with or without PNF has been used in practice because PNFs alone cannot provide complete CSF leakage repair ${ }^{25,33,37,44,46,53,56)}$. Various reconstruction methods were made by combining autologous tissues, such as fascia, fat, cartilage, and bone, various allogenic and artificial materials, and CSF leakage was reported ranging from 0 to $25 \%$, comparing with $1.7 \%$ of this study $^{52)}$.

The reconstruction techniques with a multilayer method could be categorized as in- or onlay reconstruction by the relative location of the substitutes to the dura or rigid or nonrigid by the hardness of the substitute. For inlayer reconstruction, a sufficient amount of the free margin dural or bony structures was required to suspend it. Another concern regarding the inlayer technique was the possibility of neural compression by the intradural material because the intraoperative location and shape of neural structures are not normal and are compressed by the tumor or stretched by the intraoperative CSF leakage. There were few reports about neurovascular injury by the packed inlay graft. Still, in many previous studies, it is estimated that the position of inlay graft is stably maintained as the weight of the brain pushes the graft to outward direction after intraoperative CSF leakage and postoperative postural change ${ }^{21,58)}$. Therefore, although there has been no report about neurovascular compression by inlay graft yet, in cases of sellar or parasellar locations that is a cluster of microscopic neurovascular structures, neural compression by the reconstruction material can occur with the returning process of neural tissues in the postoperative period, even though it does not occur during the operation. These difficulties of layer re- construction frequently occur after resection of the tuberculum sellae invading the optic canal. In contrast, onlay reconstruction requires only the free outer dura or bony margin and is associated with a small risk for intradural compression. The need for rigid reconstruction in ESS has been debated because it can be associated with a risk of extrusion of the reconstruction materials, chronic inflammation caused by its avascular feature and mechanical compression of the neural structures in the indented skull base area with the gasket-seal technique ${ }^{7,18,22)}$. However, the benefits of rigid reconstruction seem to be evident, as it serves as a protective barrier, preventing postoperative brain herniation regardless of intracranial pressure or brain damage during the manipulation in the nasal cavity ${ }^{7,17,23)}$.

Regarding the reconstruction materials, autologous grafts have great advantages, such as biocompatibility, resistance to infection, and long-term safety, and they are cost-free ${ }^{22,27,42,59)}$. However, the materials can be associated with harvest site complications due to the additional surgical incision, and the resources to be harvested may be limited in terms of number and size ${ }^{42)}$. In addition, reconstruction with autologous materials requires a long period of time to achieve completely watertight reconstruction because it is based on the healing process, such as fibrosis, except for when it is performed with direct dural sutures. The most pressing concern is that the CSF leakage rate is as high as 5\%, even with CSF diversion, but its beneficial effects have been shown by a prospective, randomized controlled study ${ }^{8,64)}$. The use of direct dural sutures with or without substitutes is a unique surgical technique performed to provide immediate CSF leakage repair; however, this technique has been attempted mostly in pituitary surgery, which has relatively large intradural dead space, wide dural margin and bony window due to sellar enlargement, and it requires special surgical instruments, long training procedures and long operation times ${ }^{2,3,19)}$. Therefore, the advantages of artificial substitutes, the prevention of complications related to the harvest site, a shorter time to achieve a complete watertight status and the availability of unlimited resources in the amount or design, cannot be overlooked.

Ideal reconstruction materials for ESS should be easy to manipulate in deep surgical fields, compatible with MR images, inert to host reactions, and unlimited to be acquired or designed. Therefore, we adopted the FSP and HAC as the inner and intermediate layer in the multilayer reconstruction, re- 
spectively, which are commercially available, safe and widely used in the field of neurosurgical procedures, and covered the $\mathrm{HAC}$ with the $\mathrm{PNF}^{1,20,40)}$. The purpose of each layer resembles the three protective barriers of the brain, which are composed of the dura, skull and scalp. In addition, this concept is the same as that in the layer-by layer reconstruction method of duro-cranioplasty with a rotating scalp flap in transcranial approaches. FSP has been commonly used as a substitute in duroplasty for the prevention of CSF leakage in the transcranial approach and TSA, as it has a reliable repair rate and is safe ${ }^{11,26,28,31,57,60)}$. However, it did not provide complete repair of the high-flow CSF leakage. Therefore, we considered it as the first and temporary barrier to CSF and reinforced it with material to achieve watertight reconstruction with HAC. HAC paste has been used for CSF leakage repair with rigid reconstruction for the anterior and lateral skull base ${ }^{1,5,6,40,41)}$. The use of HAC in TSA has been attempted with the introduction of injectable HAC because of the deep location of the surgical field with a narrow window. The objectives of using HAC in TSA were to sustain or protect the dura layer with the rigidity of the HAC or to achieve a CSF leakage repair, and these methods showed reliable results ${ }^{4,12,37)}$. There is a debate about whether rigid reconstruction is necessary for ESS to prevent brain herniation, but there have been few reports ${ }^{7)}$. Eloy et al. ${ }^{17)}$ succeeded in reconstruction without CSF leakage after anterior skull base tumor resection by ESS in all nine patients using consecutive onlay of autologous fascia lata, acellular dermal allograft, and PNF, nevertheless CSF leakage of the anterior skull base is not high-flow. However, the anterior skull base is not the location where high-flow CSF leakage occurs. For high-flow CSF leakage, it is reasonable to use HAC to make immediately watertight until delayed waterproofing by PNF. However, the neo-mucosalization on the exposed HAC in the nasal cavity has been questioned. Therefore, we added the PNF over the HAC and surrounding skull base bone to prevent a crust from forming on the HAC and CSF leakage. However, we experienced a 1.7\% CSF leakage rate due to technical errors from the uncovered skull base bone, as there was a small PNF that did not graft on the whole skull base bone around the HAC. Afterwards, we rechecked the margin of the HAC after hardening.

Another advantage of our reconstruction technique is that it does not require the CSF diversion, such as lumbar drainage. No restriction on position and ambulation was necessary from right after surgery. For the transtuberculum and transplanum approach, the majority of approach in our subjects, many simpler methods than ours have shown good outcomes in terms of CSF leakage ${ }^{9,16,34,37)}$. However, CSF diversion was necessary for most of the other methods. There has been still debate that PNF necrosis is increased by HAC, but in most cases, PNF necrosis is thought to be caused by torsion of pedicle or infection ${ }^{10,43,45)}$. In the cases of autologous bone graft used for solid support instead of HAC, the lumbar drain is required because it is not immediately watertight, unlike HAC. Therefore, we were able to avoid side effects related with the lumbar drainage or long-time bed rest.

The surgical field of ESS is regarded as a clean contaminated wound, and the risk of infection is inevitably higher than that in clean wounds. High-flow CSF leakage carries a higher risk of infection than low-flow leakage due to the lack of physical barriers, such as diaphragma sellae, and difficulty in reconstruction. There were three atypical clinical features in our results. First, the infection rate of this technique was as high as $13.5 \%$, which was higher than the $1.7 \%$ CSF leakage rate and much higher than the $3 \%$ infection rate in temporal bone reconstruction using $\mathrm{HAC}^{40)}$. This finding was different from those in previous studies showing a similar or lower ratio of the infection rate to the CSF leakage rate with ESS and highflow CSF leakage ${ }^{13,36,39,62)}$. The second feature was that no microorganism was cultured in the CSF specimens. Of course, we could not verify that the negative culture results always corresponded to aseptic meningitis because the isolation rate of the CSF culture was approximately $33 \%$ and perioperative antibiotics influenced the culture results ${ }^{15,30)}$. The third one was that the time to infection was longer than 8 days (range, 8-38) after the operation in half of the infected patients, which was longer than that of postcraniotomy meningitis. We speculated that these findings were related to the amount of inoculated microorganisms and the inflammation reaction to the foreign body. The FSP and HAC used in the study had innate defects that were susceptible to infection, such as other allogenic materials, and microbiological contamination of the materials appears inevitable in the clean contaminated passage of the nasal cavity. The amount of microorganisms, not enough to cause the meningitis when using an autologous materials, could manifest the overt meningitis after the incubation period in the avascular allogenic material. Therefore, the different amounts of inoculated microorganisms across 
individuals in the intracranial space might determine the outbreak and timing of overt presentation, even though the same preparation and preoperative antibiotics were applied. We suppose that a more precise diagnostic method of bacterial meningitis based on molecular biological techniques can verify our presumption ${ }^{49)}$. The inflammatory reaction after reconstruction with HAC has been documented in transcranial approaches ${ }^{5,40,47,61)}$. A pathohistological study on surgical specimen in complicated cases revealed a foreign body inflammatory reaction showing fibrosis, granulation of the tissue, and the presence of neutrophils, lymphocytes, and plasma cell around the grafted material $^{48,50)}$. A large number of plasma cells in the surrounding tissue might indicate not only inflammation but also the immune response to material itself ${ }^{50)}$. The development of biologically inert materials for watertight rigid reconstruction is mandatory, and stronger materials than FSP may be required to prevent some materials of HXA from influx into the intracranial space.

This study from three institutions had an inherent limitation, as it is a retrospective study, and the results can be biased by the different surgical experiences of the surgeons. However, we introduced a technique that realizes immediate CSF leakage repair and complete skull base healing with commercially available allogenic materials after EES.

\section{CONCLUSION}

Our reconstruction technique was easy to implement and provided immediate watertight skull base reconstruction with a reliable repair rate for high-flow CSF leakage in EES, but it was accompanied by an unavoidable risk of infection. Our results showed that the development of more biologically compatible allogenic material is required.

\section{CONFLICTS OF INTEREST}

No potential conflict of interest relevant to this article was reported.

\section{INFORMED CONSENT}

This type of study does not require informed consent.

\section{AUTHOR CONTRIBUTIONS}

\author{
Conceptualization : YHK, HK, YSD, KH, JDJ, YHK \\ Data curation : YHK, YSD, KH, JDJ, YHK \\ Formal analysis : YHK, YHK \\ Funding acquisition : $\mathrm{HK}$, YHK \\ Methodology : YHK, YHK \\ Project administration : YHK, HK, KH, YHK \\ Visualization: YHK, YHK \\ Writing - original draft : YHK, YHK \\ Writing - review \& editing: YHK, HK, YSD, KH, JDJ, \\ YHK
}

\section{ORCID}

Young-Hoon Kim https://orcid.org/0000-0002-8852-6503 Ho Kang https://orcid.org/0000-0003-2143-410X

Yun-Sik Dho https://orcid.org/0000-0001-5505-0812

Kihwan Hwang https://orcid.org/0000-0001-7211-729X

Jin-Deok Joo https://orcid.org/0000-0002-0728-3336

Yong Hwy Kim https://orcid.org/0000-0001-9009-4191

\section{- Acknowledgements}

This study is supported by a grant (No. 0520200030 to Kim YH) from Seoul National University Hospital.

Authors would like to express our deepest appreciation to Mijin Jung for her support of medical illustrations.

\section{- Supplementary materials}

The online-only data supplement is available with this article at https://doi.org/10.3340/jkns.2020.0231.

\section{References}

1. Abe T, Anan M, Kamida T, Fujiki M : Surgical technique for anterior skull base reconstruction using hydroxyapatite cement and titanium mesh. 
Acta Neurochir (Wien) 151 : 1337-1338, 2009

2. Acerbi F, Genden E, Bederson J : Circumferential watertight dural repair using nitinol U-clips in expanded endonasal and sublabial approaches to the cranial base. Neurosurgery 67(2 Suppl Operative) : 448-456, 2010

3. Ahn JY, Kim SH : A new technique for dural suturing with fascia graft for cerebrospinal fluid leakage in transsphenoidal surgery. Neurosurgery 65(6 Suppl) : 65-71; discussion 71-72, 2009

4. Alvarez Berastegui GR, Raza SM, Anand VK, Schwartz TH : Endonasal endoscopic transsphenoidal chiasmapexy using a clival cranial base cranioplasty for visual loss from massive empty sella following macroprolactinoma treatment with bromocriptine: case report. J Neurosurg 124 : 1025-1031, 2016

5. Arriaga MA, Chen DA : Hydroxyapatite cement cranioplasty in translabyrinthine acoustic neuroma surgery. Otolaryngol Head Neck Surg $126:$ 512-517, 2002

6. Ascherman JA, Foo R, Nanda D, Parisien M : Reconstruction of cranial bone defects using a quick-setting hydroxyapatite cement and absorbable plates. J Craniofac Surg 19 : 1131-1135, 2008

7. Battaglia P, Turri-Zanoni M, Castelnuovo P, Prevedello DM, Carrau RL : Brain herniation after endoscopic transnasal resection of anterior skull base malignancies. Neurosurgery 11 Suppl 3 : 457-462; discussion 462, 2015

8. Caggiano C, Penn DL, Laws ER Jr : The role of the lumbar drain in endoscopic endonasal skull base surgery: a retrospective analysis of 811 cases. World Neurosurg 117 : e575-e579, 2018

9. Cavallo LM, Messina A, Esposito F, de Divitiis O, Dal Fabbro M, de Divitiis $E$, et al. : Skull base reconstruction in the extended endoscopic transsphenoidal approach for suprasellar lesions. J Neurosurg 107 : 713-720, 2007

10. Chabot JD, Patel CR, Hughes MA, Wang EW, Snyderman CH, Gardner PA, et al. : Nasoseptal flap necrosis: a rare complication of endoscopic endonasal surgery. J Neurosurg 128 : 1463-1472, 2018

11. Chauvet D, Tran V, Mutlu G, George B, Allain JM : Study of dural suture watertightness: an in vitro comparison of different sealants. Acta Neurochir (Wien) $153:$ 2465-2472, 2011

12. Chung SB, Nam DH, Park K, Kim JH, Kong DS : Injectable hydroxyapatite cement patch as an on-lay graft for the sellar reconstructions following endoscopic endonasal approach. Acta Neurochir (Wien) 154 : 659664; discussion 664, 2012

13. Conger A, Zhao F, Wang X, Eisenberg A, Griffiths C, Esposito F, et al. : Evolution of the graded repair of CSF leaks and skull base defects in endonasal endoscopic tumor surgery: trends in repair failure and meningitis rates in 509 patients. J Neurosurg $130: 861-875,2018$

14. Dho YS, Kim YH, Se YB, Han DH, Kim JH, Park CK, et al. : Endoscopic endonasal approach for craniopharyngioma: the importance of the relationship between pituitary stalk and tumor. J Neurosurg 129 : 611619, 2018

15. Druel B, Vandenesch F, Greenland T, Verneau V, Grando J, Salord F, et al. : Aseptic meningitis after neurosurgery: a demonstration of bacterial involvement. Clin Microbiol Infect 1 : 230-234, 1996
16. Dusick JR, Esposito F, Kelly DF, Cohan P, DeSalles A, Becker DP, et al. : The extended direct endonasal transsphenoidal approach for nonadenomatous suprasellar tumors. J Neurosurg 102 : 832-841, 2005

17. Eloy JA, Shukla PA, Choudhry OJ, Singh R, Liu JK : Assessment of frontal lobe sagging after endoscopic endonasal transcribriform resection of anterior skull base tumors: is rigid structural reconstruction of the cranial base defect necessary? Laryngoscope 122 : 2652-2657, 2012

18. Garcia-Navarro V, Anand VK, Schwartz TH : Gasket seal closure for extended endonasal endoscopic skull base surgery: efficacy in a large case series. World Neurosurg 80 : 563-568, 2013

19. Gardner P, Kassam A, Snyderman C, Mintz A, Carrau R, Moossy JJ : Endoscopic endonasal suturing of dural reconstruction grafts: a novel application of the U-Clip technology. Technical note. J Neurosurg 108 : 395-400, 2008

20. George B, Matula C, Kihlström L, Ferrer E, Tetens V : Safety and efficacy of TachoSil (absorbable fibrin sealant patch) compared with current practice for the prevention of cerebrospinal fluid leaks in patients undergoing skull base surgery: a randomized controlled trial. Neurosurgery 80 : 847-853, 2017

21. Germani RM, Vivero R, Herzallah IR, Casiano RR : Endoscopic reconstruction of large anterior skull base defects using acellular dermal allograft. Am J Rhinol 21 : 615-618, 2007

22. Gil Z, Abergel A, Leider-Trejo L, Khafif A, Margalit N, Amir A, et al. : A comprehensive algorithm for anterior skull base reconstruction after oncological resections. Skull Base 17 : 25-37, 2007

23. Hanna AS, Grindle CR, Patel AA, Rosen MR, Evans JJ : Inadvertent insertion of nasogastric tube into the brain stem and spinal cord after endoscopic skull base surgery. Am J Otolaryngol 33 : 178-180, 2012

24. Harvey RJ, Parmar P, Sacks R, Zanation AM : Endoscopic skull base reconstruction of large dural defects: a systematic review of published evidence. Laryngoscope 122 : 452-459, 2012

25. Hasegawa H, Shin M, Kondo K, Saito N : Reconstruction of dural defects in endoscopic transnasal approaches for intradural lesions using multilayered fascia with a pressure-control spinal drainage system. World Neurosurg 114 : e1316-e1324, 2018

26. Heymanns V, Oseni AW, Alyeldien A, Maslehaty H, Parvin R, Scholz M, et al. : Sandwich wound closure reduces the risk of cerebrospinal fluid leaks in posterior fossa surgery. Clin Pract 6 : 824, 2016

27. Hoffmann TK, El Hindy N, Müller OM, Schuler PJ, Bergmann C, Hierner R, et al. : Vascularised local and free flaps in anterior skull base reconstruction. Eur Arch Otorhinolaryngol 270 : 899-907, 2013

28. Hong CK, Kim YB, Hong JB, Lee KS : Sealing of cerebrospinal fluid leakage during conventional transsphenoidal surgery using a fibrin-coated collagen fleece. J Clin Neurosci 22 : 696-699, 2015

29. Hong SD, Nam DH, Kong DS, Kim HY, Chung SK, Dhong HJ : Endoscopic modified transseptal transsphenoidal approach for maximal preservation of sinonasal quality of life and olfaction. World Neurosurg 87 : 162169, 2016

30. Huang B, Ren Y, Wang C, Lan Z, Hui X, Liu W, et al. : Risk factors for postoperative meningitis after microsurgery for vestibular schwannoma. PLoS One 14 : e0217253, 2019 
31. Hutter $G$, von Felten $S$, Sailer MH, Schulz M, Mariani L : Risk factors for postoperative CSF leakage after elective craniotomy and the efficacy of fleece-bound tissue sealing against dural suturing alone: a randomized controlled trial. J Neurosurg 121 : 735-744, 2014

32. Ishikawa T, Takeuchi K, Nagata Y, Choo J, Kawabata T, Ishizaki T, et al. : Three types of dural suturing for closure of CSF leak after endoscopic transsphenoidal surgery. J Neurosurg 131 : 1625-1631, 2019

33. Jeon C, Hong SD, Seol HJ, Lee JI, Nam DH, Hwang YJ, et al. : Reconstructive outcome of intraoperative cerebrospinal fluid leak after endoscopic endonasal surgery for tumors involving skull base. J Clin Neurosci 45 : 227-231, 2017

34. Kaptain GJ, Vincent DA, Laws ER Jr : Cranial base reconstruction after transsphenoidal surgery with bioabsorbable implants. Neurosurgery 48 : 232-233; discussion 233-234, 2001

35. Kassam AB, Prevedello DM, Carrau RL, Snyderman CH, Thomas A, Gardner $\mathrm{P}$, et al. : Endoscopic endonasal skull base surgery: analysis of complications in the authors' initial 800 patients. J Neurosurg 114 : 1544-1568, 2011

36. Kim YH, Jeon C, Se YB, Hong SD, Seol HJ, Lee Jl, et al. : Clinical outcomes of an endoscopic transclival and transpetrosal approach for primary skull base malignancies involving the clivus. J Neurosurg 128 : 1454-1462, 2018

37. Kitano M, Taneda M : Icing and multilayering technique of injectable hydroxyapatite cement paste for cranial base reconstruction after transsphenoidal surgery: technical note. Neurosurgery 61(3 Suppl) : E53E54; discussion E54, 2007

38. Kong DS, Hong CK, Hong SD, Nam DH, Lee Jl, Seol HJ, et al. : Selection of endoscopic or transcranial surgery for tuberculum sellae meningiomas according to specific anatomical features: a retrospective multicenter analysis (KOSEN-002). J Neurosurg 130 : 838-847, 2018

39. Koutourousiou M, Fernandez-Miranda JC, Stefko ST, Wang EW, Snyderman CH, Gardner PA : Endoscopic endonasal surgery for suprasellar meningiomas: experience with 75 patients. J Neurosurg 120 : 13261339, 2014

40. Kveton JF, Coelho DH : Hydroxyapatite cement in temporal bone surgery: a 10 year experience. Laryngoscope $114: 33-37,2004$

41. Kveton JF, Goravalingappa R : Elimination of temporal bone cerebrospinal fluid otorrhea using hydroxyapatite cement. Laryngoscope 110(10 Pt 1) : 1655-1659, 2000

42. Lam K, Luong AU, Yao WC, Citardi MJ : Use of autologous fat grafts for the endoscopic reconstruction of skull base defects: indications, outcomes, and complications. Am J Rhinol Allergy 32 : 310-317, 2018

43. Lavigne P, Faden DL, Wang EW, Snyderman CH : Complications of nasoseptal flap reconstruction: a systematic review. J Neurol Surg B Skull Base 79(Suppl 4) : S291-S299, 2018

44. Leng LZ, Brown S, Anand VK, Schwartz TH : "Gasket-seal" watertight closure in minimal-access endoscopic cranial base surgery. Neurosurgery 62(5 Suppl 2) : ONSE342-ONSE343; discussion ONSE343, 2008

45. Liu JK, Schmidt RF, Choudhry OJ, Shukla PA, Eloy JA : Surgical nuances for nasoseptal flap reconstruction of cranial base defects with high-flow cerebrospinal fluid leaks after endoscopic skull base surgery. Neuro- surg Focus $32: E 7,2012$

46. Luginbuhl AJ, Campbell PG, Evans J, Rosen M : Endoscopic repair of high-flow cranial base defects using a bilayer button. Laryngoscope $120: 876-880,2010$

47. Luryi AL, Schutt CA, Michaelides E, Kveton JF : Hydroxyapatite cement cranioplasty for translabyrinthine surgery: a single institution experience. Laryngoscope $130: 206-211,2020$

48. Matic $D$, Phillips JH : A contraindication for the use of hydroxyapatite cement in the pediatric population. Plast Reconstr Surg $110: 1-5,2002$

49. Moon J, Kim N, Kim TJ, Jun JS, Lee HS, Shin HR, et al. : Rapid diagnosis of bacterial meningitis by nanopore $16 \mathrm{~S}$ amplicon sequencing: a pilot study. Int J Med Microbiol 309 : 151338, 2019

50. Moreira-Gonzalez A, Jackson IT, Miyawaki T, Barakat K, DiNick V : Clinical outcome in cranioplasty: critical review in long-term follow-up. J Craniofac Surg 14 : 144-153, 2003

51. Moussazadeh N, Prabhu V, Bander ED, Cusic RC, Tsiouris AJ, Anand VK, et al. : Endoscopic endonasal versus open transcranial resection of craniopharyngiomas: a case-matched single-institution analysis. Neurosurg Focus 41 : E7, 2016

52. Oakley GM, Christensen JM, Winder M, Jonker BP, Davidson A, Steel T, et al. : Collagen matrix as an inlay in endoscopic skull base reconstruction. J Laryngol Otol 132 : 214-223, 2018

53. Park JH, Choi JH, Kim Yl, Kim SW, Hong YK : Modified graded repair of cerebrospinal fluid leaks in endoscopic endonasal transsphenoidal surgery. J Korean Neurosurg Soc 58 : 36-42, 2015

54. Phi JH, Kim YH, Kim JH, Kim DG : Clinical and anatomic features of supraglandular pituitary adenomas. World Neurosurg 92 : 241-248, 2016

55. Qiao N : Endocrine outcomes of endoscopic versus transcranial resection of craniopharyngiomas: a system review and meta-analysis. Clin Neurol Neurosurg 169 : 107-115, 2018

56. Shorter CD, Connor DE Jr, Thakur JD, Gardner G, Nanda A, Guthikonda $B$ : Repair of middle fossa cerebrospinal fluid leaks using a novel combination of materials: technical note. Neurosurg Focus 32 : E8, 2012

57. So J, Park H, Sung KS, Lee KS, Hong CK : Sandwich technique using fibrin-coated collagen fleece for sellar reconstruction in large dural defects during transsphenoidal surgery. J Clin Neurosci 43 : 256-260, 2017

58. Soudry E, Turner JH, Nayak JV, Hwang PH : Endoscopic reconstruction of surgically created skull base defects: a systematic review. Otolaryngol Head Neck Surg $150:$ 730-738, 2014

59. Tabaee A, Anand VK, Brown SM, Lin JW, Schwartz TH : Algorithm for reconstruction after endoscopic pituitary and skull base surgery. Laryngoscope 117 : 1133-1137, 2007

60. Tews J, Jahromi BR, Ludtka C, Schwan S, Ota N, Meisel HJ, et al. : TachoSil dural reconstruction in extracranial-intracranial bypass surgeries. J Neurol Surg A Cent Eur Neurosurg 80 : 39-43, 2019

61. Volsky PG, Hillman TA, Stromberg KJ, Buchinsky FJ, Chen DA, Jackson $N M$, et al. : Hydroxyapatite cement cranioplasty following translabyrinthine approach: long-term study of 369 cases. Laryngoscope 127 : 2120-2125, 2017 
62. Yamada S, Fukuhara N, Yamaguchi-Okada M, Nishioka H, Takeshita A, Takeuchi $Y$, et al. : Therapeutic outcomes of transsphenoidal surgery in pediatric patients with craniopharyngiomas: a single-center study. J Neurosurg Pediatr 21 : 549-562, 2018

63. Zwagerman NT, Geltzeiler MN, Wang EW, Fernandez-Miranda JC, Snyderman $\mathrm{CH}$, Gardner PA : Endonasal suturing of nasoseptal flap to nasopharyngeal fascia using the V-Loc ${ }^{\mathrm{TM}}$ wound closure device: 2-di- mensional operative video. Oper Neurosurg (Hagerstown) $16: 40$ 41, 2019

64. Zwagerman NT, Wang EW, Shin SS, Chang YF, Fernandez-Miranda JC,

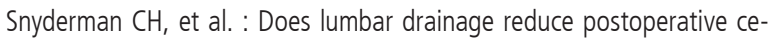
rebrospinal fluid leak after endoscopic endonasal skull base surgery? A prospective, randomized controlled trial. J Neurosurg 131 : 1172-1178, 2019 